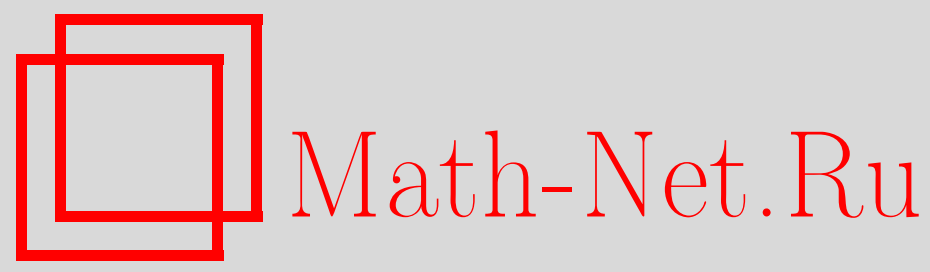

Д. В. Болотов, Гиперслоения на компактных трехмерных многообразиях с ограничениями на внешнюю кривизну слоев, Матем. заметки, 1998, том 63, выпуск 5, 651-659

DOI: https://doi.org/10.4213/mzm1330

Использование Общероссийского математического портала Math-Net.Ru подразумевает, что вы прочитали и согласны с пользовательским соглашением http://www.mathnet.ru/rus/agreement

Параметры загрузки:

IP : 54.157 .27 .8

26 апреля 2023 г., 18:33:28

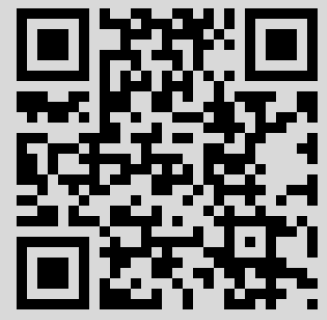




\title{
ГИПЕРСЛОЕНИЯ НА КОМПАКТНЫХ ТРЕХМЕРНЫХ МНОГООБРАЗИЯХ С ОГРАНИЧЕНИЯМИ \\ НА ВНЕШНЮЮ КРИВИЗНУ СЛОЕВ
}

\section{Д. В. Болотов}

\begin{abstract}
Исследуются $C^{\infty}$-слоения коразмерности 1 на компактных трехмерных римановых многообразиях. Вводятся новые классы слоений, а именно, гиперболические, параболические и эллиптические. Приводятся примеры таких слоений. В частности, строится метрика класса $C^{\infty}$ неотрицательной секционной кривизны на $S^{3}$ такая, что слоение Риба является параболическим для этой метрики. Классифицируются аналитические трехмерные компактные многообразия знакопостоянной секционной кривизны, допускающие параболические слоения.
\end{abstract}

Библиография: 10 названий.

1. Введение. Пусть $\left(M, g_{M}\right)$ - риманово многообразие и $\mathscr{F}$ - слоение на нем. Многообразие и слоение на нем везде предполагаются класса $C{ }^{\infty}$. Обозначим через $L$ подрасслоение касательного расслоения $T M$, касательное к $\mathscr{F}$, а через $Q$ - нормальное расслоение, которое есть фактор $T M / L$. Тогда имеет место точная расщепляющаяся последовательность расслоений:

$$
0 \rightarrow L \rightarrow T M \rightarrow Q \rightarrow 0
$$

Скажем, что слоение ориентируемо, если ориентируемо $L$, и трансверсально ориентируемо, если ориентируемо $Q$.

Пусть $\sigma: Q \rightarrow L^{\perp} \subset T M$ - расщепляющий изоморфизм, где $L^{\perp}-$ подрасслоение $T M$, ортогональное к $L$. Представим $g_{M}$ в виде $g_{M}=g_{L} \oplus g_{L} \perp$. Положим $g_{Q}=\sigma^{*} g_{L}$. Тогда $\sigma$ есть метрический изоморфизм расслоений $\left(Q, g_{Q}\right)$ и $\left(L, g_{L} \perp\right)$, которые мы в дальнейшем будем отождествлять.

Определим вторую фундаментальную форму слоения, как отображение $\alpha$, положив $\alpha\left(X, X^{\prime}\right)=\pi\left(\nabla_{X} X^{\prime}\right)$, где $X, X^{\prime}$ - гладкие сечения $L$, a $\nabla$ - метрическая связность на $M$. Определим оператор второй фундаментальной формы слоения $A_{Z}: L \rightarrow L$ для сечения $Z$ расслоения $L^{\perp}$ из соотношения $g_{M}\left(\alpha\left(X, X^{\prime}\right), Z\right)=g_{M}\left(A_{Z} X, X^{\prime}\right)$. Так как $g_{M}\left(\nabla_{X} X^{\prime}, Z\right)=-g_{M}\left(X^{\prime}, \nabla_{X} Z\right)$, то видно, что $A_{Z} X=-\pi^{\perp} \nabla_{X} Z$, где $\pi^{\perp}: T M \rightarrow L-$ естественная проекция. Пусть теперь $\mathscr{F}$ - ориентируемое гиперслоение на $M$, а $\xi$ - единичное, ортогональное к $\mathscr{F}$ векторное поле. В этом случае оператор $A_{\xi}$ назовем оператором второй квадратичной формы слоения $\mathscr{F}$. Из того, что $g_{M}\left(\nabla_{X} \xi, \xi\right)=0$, следует, что $A_{\xi}=-\nabla_{X} \xi$. 


\section{2. Предварительные сведения.}

Теорема 1 [1]. Пусть $F-C^{\infty}$-ориентируемое слоение на компактном многообразии $W^{n+1}$ постоянной кривизны $c$.

Пусть $K_{F}: W^{n+1} \rightarrow \mathbb{R}$ гладкая функиия такая, что $K_{F}(x)$ - гауссова кривизна слоя $L$, проходящего через $x$, а $\mu$-канонический обвем на $W^{n+1}$. Определим $\overline{K_{F}}$ из равенства

$$
\operatorname{Vol}\left(W^{n+1}\right) \overline{K_{F}}=\int_{W^{n+1}} K_{F} \mu .
$$

Тогда имеет место следующая формула:

$$
\overline{K_{F}}= \begin{cases}2^{n} c^{n / 2} /\left(\begin{array}{c}
n \\
n / 2
\end{array}\right) & \text { для четного } n, \\
0 & \text { для нечетного } n\end{cases}
$$

Брито, Ланжевин, Розенберг в [2] обобщили этот результат и доказали следующую теорему.

Теорема 2. Пусть $W^{n+1}$ и F такие, как выше, а $\sigma_{i}$ - әлементарные симметрические многочлены от собственных значений оператора второй квадратичной формы $A_{\xi}$ слоения. Тогда верна следующая формула:

$$
\int_{W^{n+1}} \sigma_{i} \mu= \begin{cases}\left(\begin{array}{l}
n / 2 \\
i / 2
\end{array}\right) c^{i / 2} \mathrm{Vol} W^{n+1}, & \text { если } n, i \text { четные } \\
0 & \text { в остальных случаях }\end{cases}
$$

ЗАмЕчАниЕ 1 . В случае $i=1$ без предположения постоянства секционной кривизны $W^{n+1}$ имеем $\int_{W^{n+1}} \sigma_{1} \mu=0$ [3].

Заметим, что в случае $n=2 \sigma_{2}$ имеет наглядньй геометрический смысл: $\sigma_{2}(x)=$ $K^{F}(x)-K_{\sigma}^{F}(x)=K_{e}^{F}(x)$ (уравнение Гаусса) есть внешняя кривизна слоя $K_{e}^{F}(x)$, проходящего через $x$, а именно, разность секционных кривизн слоя $K^{F}(x)$ и объемлющего пространства $K_{\sigma}^{F}(x)$ вдоль площадок, касательных к слою.

Теорема 3 [4]. Пусть $\mathscr{F}$ - гладкое слоение на замкнутом ориентируемом трехмерном многообразии $M^{3}$, причем все слои гомеоморфны $\mathbb{R}^{2}$. Тогда $M^{3}$ гомеоморфно тору.

Теорема 4 [5]. Пусть $M^{n}$ - компактное риманово многообразие типа $K(\pi, 1)$. Тогда если секиионная кривизна $M^{n}$ неотрицательна, то она тохдественно равна 0 .

ТеОрема 5 [6]. Пусть $M^{n}$ - полное риманово многообразие с постоянным нульиндексом $\mu=k>0$. Тогда если существует поверхность, ортогональная слоям вполне геодезического слоения, определяемого нуль-индексом, то $M^{n}$ локально есть метрическое произведение риманова многообразия $M^{n-k}$ и евклидова пространства $E^{k}$.

Напомним определения некоторых классов слоений, интенсивно исследуемых в последнее время. 
ОПРЕДЕлЕниЕ 1. Слоение на $M$ называется вполне геодезическим, если слои вполне геодезические подмногообразия $M$. Это равносильно тому, что $\alpha \equiv 0$, что эквивалентно условию $\Theta(Z) g_{L}=0$ для любого сечения $Z$ расслоения $L^{\perp}$, где $\Theta(Z)-$ производная Ли в направлении векторного поля $Z$.

ОПРЕДЕЛЕНИЕ 2. Слоение на $M$ называется $p$ имановым, если $\Theta(X) g_{L_{\perp}}=0$ для любого сечения $X$ расслоения $L$. То есть слоение локально является римановой субмерсией.

ОПРЕДЕЛЕниЕ 3. Слоениена $M$ называется гармоническим, если слои-минимальные подмногообразия $M$, что эквивалентно условию $\operatorname{trace}\left(A_{Z}\right)=0$ для любого сечения $Z$ расслоения $L_{\perp}$.

ОПРЕДЕЛЕНИЕ 4. Слоение на $M$ называется вполне омбилическим, если слои суть вполне омбилические подмногообразия $M$.

Следуюшие определения принадлежат профессору А. А. Борисенко.

ОПРЕДЕЛЕНИЕ А. Параболическим слоением назьвается слоение, для которого $K_{e}^{F} \equiv 0$.

ОПРЕДЕЛЕНИЕ В. Гиперболическим слоением называется слоение, для которого $K_{e}^{F} \leqslant 0$ и которое не является параболическим. В случае строгого неравенства скажем, что слоение - сильно гиперболическое. (Заметим, что в трехмерном случае гармоническое слоение является гиперболическим).

ОПРЕДЕЛЕНИЕ С. Эллиптическим слоением назьвается слоение, для которого $K_{e}^{F} \geqslant 0$ и которое не является параболическим. (В трехмерном случае примером эллиптического слоения является вполне омбилическое слоение).

ЗАмЕчАниЕ 2. Любое компактное трехмерное риманово многообразиене допускает сильно эллиптического слоения и параболического слоения с $\operatorname{rank} A_{\xi} \equiv 1$. Это прямое следствие замечания 1 .

\section{3. Формулировки и доказательства основных результатов.}

Теорема А. Пусть $M^{3}$ - компактное ориентируемое риманово многообразие и F - ориентируемое слоение коразмерности 1 на нем, а $\xi$ - единичное, ортогональное к $\mathscr{F}$ векторное поле. Тогда верна следующая формула:

$$
\int_{M^{3}} \operatorname{Ric}(\xi, \xi) \mu=2 \int_{M^{3}} K_{e}^{\mathscr{F}} \mu,
$$

әде $\mu$ - форма обгема на $M^{3}$, a $\operatorname{Ric}(\xi, \xi)$ - кривизна Риччи в направлении $\xi$.

СлЕДСТВИЕ. Если компактное трехмерное риманово многообразие $M^{3}$ является многообразием неотричательной кривизны Риччи, то оно не допускает гиперболического слоения. Если компактное трехмерное риманово многообразие $M^{3}$ является многообразием неполохсительной кривизны Риччи, то оно не допускает әллиптического слоения. Если компактное трехмерное риманово многообразие $M^{3}$ является многообразием знакопостоянной кривизны Риччи, то оно не допускает параболического слоения. 
ЗАмЕчАниЕ 3 . В случае неориентируемости $M^{3}$ или $\mathscr{F}$ следует перейти к ориентируемому конечнолистному накрытию $\mathscr{M}^{3}$ с индуцированной метрикой и ориентируемьм поднятьм слоением $\Phi$. Таким образом, следствия остаются верными и в неориентируемом случае.

Теорему А можно вьвести из [7]. Однако в духе данной работы приведем доказательство, используя технику, используемую в [2].

ДокАЗАТЕЛЬСТво тЕОРЕМЫ А. Пусть $\left\{e_{1}, e_{2}, e_{3}\right\}$ - локальньй ортонормированный базис на $M^{3}$, причем $e_{3}$ совпадает с $\xi$. Через $\left\{\Theta_{1}, \Theta_{2}, \Theta_{3}\right\}$ обозначим двойственный базис ковекторов. Определим 1-формы $w_{i, j}(i, j=1,2,3)$ и $\psi_{k}(k=0,1,2)$ на $M^{3}$ следующим образом: $w_{i, j}(u)=\left\langle\nabla_{u} e_{i}, e_{j}\right\rangle$, где $\nabla$ - метрическая связность на $M^{3}$, а $\psi_{k}$ определяются из соотношения

$$
\sum_{k=0}^{2} \psi_{k} t^{k}=\left(t \Theta_{1}+w_{13}\right) \wedge\left(t \Theta_{2}+w_{23}\right)
$$

В [2] доказьвается, что $\psi_{k}$ не зависят от выбора базиса с той же ориентацией и такого, что $e_{3}=\xi$.

УТВЕРЖДЕНИЕ. $d \psi_{1}=2 \psi_{0} \wedge \Theta_{3}-\operatorname{Ric}(\xi, \xi) \mu$.

ДокАЗАТЕЛЬСТво. Имеем

$$
\begin{aligned}
& d\left(w_{13} \wedge \Theta_{2}+\Theta_{1} \wedge w_{23}\right)=d w_{13} \wedge \Theta_{2}-w_{13} \wedge d \Theta_{2}+d \Theta_{1} \wedge w_{23}-\Theta_{1} \wedge d w_{23} \\
& =d w_{13} \wedge \Theta_{2}+w_{13} \wedge \Theta_{1} \wedge w_{21}+w_{13} \wedge \Theta_{3} \wedge w_{23} \\
& \quad-\Theta_{2} \wedge w_{12} \wedge w_{23}-\Theta_{3} \wedge w_{13} \wedge w_{23}-\Theta_{1} \wedge d w_{23} \\
& =d w_{13} \wedge \Theta_{2}+\Theta_{1} \wedge w_{21} \wedge w_{13}-2 \Theta_{3} \wedge w_{13} \wedge w_{23}-\Theta_{2} \wedge w_{12} \wedge w_{23}-\Theta_{1} \wedge d w_{23} \\
& =d w_{13} \wedge \Theta_{2}+\Theta_{1} \wedge d w_{23}+\Theta_{1} \wedge \Omega_{3}^{2}-2 \Theta_{3} \wedge \psi_{0}-\Theta_{2} \wedge d w_{13}-\Theta_{2} \wedge \Omega_{3}^{1}-\Theta_{1} \wedge d w_{23} \\
& =-2 \Theta_{3} \wedge \psi_{0}+\left(K_{\sigma}\left(e_{2}, \xi\right)+K_{\sigma}\left(e_{1}, \xi\right)\right) \mu=-2 \Theta_{3} \wedge \psi_{0}+\operatorname{Ric}(\xi, \xi) \mu .
\end{aligned}
$$

Утверждение доказано.

В выкладках мы использовали структурные уравнения Картана:

$$
d \Theta_{i}=-\sum_{j} \Theta_{j} \wedge w_{i j}, \quad d w_{i j}+\Omega_{j}^{i}=\sum_{k} w_{i k} \wedge w_{k j}
$$

где

$$
\Omega_{j}^{i}=\sum_{k, l} \frac{1}{2} R_{j k l}^{i} \Theta_{k} \wedge \Theta_{l}
$$

- компоненты формы кривизны многообразия $M^{3}$.

Для завершения доказательства теоремы осталось показать, что $\Theta_{3} \wedge \psi_{0}=\operatorname{det} A_{\xi} \mu$, а это в точности утверждение леммы 1.8 [2]. Таким образом, теорема А доказана. 
Теорема В. Пусть $M^{3}$ - компактное ориентируемое риманово многообразие $c$ аналитической метрикой и $\mathscr{F}$ - параболическое слоение коразмерности 1 на нем. Тогда

1) если секционная кривизна $K_{\sigma}$ многообразия неотрицательна, то либо

а) $M^{3}$ - плоская пространственная форма, либо

б) $M^{3}$ гомеоморфно $S^{2} \times S^{1}$, причем $\mathscr{F}$ в әтом случае является тривиальным $S^{2}$-расслоением над $S^{1}$, и метрика на $S^{2} \times S^{1}$ локально есть метрика прямого произведения слоя на евклидов фактор;

2) если $K_{\sigma} \leqslant 0$, то либо

а) $M^{3}$ - плоская пространственная форма, либо

б) भ्F является $M^{2}$-расслоением над $S^{1}$, где $M^{2}$ - замкнутая поверхность рода $g \geqslant 2$, и метрика на $M^{3}$ локально есть метрическое произведение слоя на евклидов фактор.

Конечнолистное накрытие $M^{3}$ гомеоморфно $M^{2} \times S^{1}$.

ЗАмечАниЕ 4. Утверждение 1 ) теоремы В не может быть обобщено на случай $C^{\infty}$ метрики (см. пример 2).

ДОКАЗАТЕЛЬСТВо ТЕОРЕМЫ В. Напомним определение внутреннего нуль-индекса точки $x$ многообразия $M$ :

$$
\mu_{x}=\operatorname{dim}\left\{X \in T_{x} M: R(X, Y)=0 \text { для любого } Y \text { из } T_{x} M\right\},
$$

где $R(X, Y)$ - оператор кривизны риманова многообразия $M$. Оказьвается, если $\mu_{x}>0$ для всех $x$, то множество $P=\left\{x: \mu_{x}=\min _{x \in M} \mu_{x}\right\}$ открыто, а распределение (1) на $P$ является интегрируемым и вполне геодезическим [8]. Непосредственно из теоремы 1 и из того, что $K_{\sigma}$ не меняет знак, следует, что $K_{\sigma}(\xi, *) \equiv 0$. Представляя тензор кривизны в точке как симметрический билинейньй оператор на трехмерном пространстве бивекторов $\Lambda^{2} T_{x} M$ и замечая (из экстремальности), что собственное подпространство $\Lambda^{2} T_{x} M$, отвечающее собственному значению 0 , двумерно, можно показать, что $R_{x}(\xi, *) \equiv 0$ для любого $x$ из $M$, т.е. $\mu_{x}=1$ или 3 . Из сказанного следует, что $P=\left\{x \in M: \mu_{x}=1\right\}$ является открытым и насыщенным (т.е. являюшимся объединением слева ортогонального слоения $\left.\mathscr{F}^{\perp}\right)$, причем слои $\mathscr{F}^{\perp}$ на $P$ являются геодезическими в $M^{3}$. Если $M^{3} \backslash P$ содержит открытое множество, то ввиду аналитичности метрики $R \equiv 0$ на $M^{3}$. Если же множество $P$ всюду плотно в $M^{3}$, то $\mathscr{F}^{\perp}$ является вполне геодезическим на всем $M^{3}$. Действительно, пусть $f: M^{3} \rightarrow \mathbb{R}$ - функция, ставящая в соответствие точке $M^{3}$ многообразия геодезическую кривизну слоя ортогонального слоения $\mathscr{F}^{\perp}$, проходящего через эту точку. Тогда $f=0$ на всюду плотном множестве $P$, поэтому $f$ есть тождественный нуль. Из теоремы 5 следует, что метрика на $M^{3}$ локально расщепляется на прямое произведение метрик, т.е. $\mathscr{F}$ является как римановым, так и вполне геодезическим. Заметим, что теорема 5 применима лишь в случае постоянства нуль-индекса в некоторой окрестности точки, однако наличие слоения позволяет перенести доказательство теоремы 5 и на особые точки. Утверждение 2) теоремы следует теперь из результатов Эберляйна [9]. Для доказательства утверждения 1) рассмотрим два случая.

Случай 1. Пусть $\mathscr{F}$ имеет компактный слой. Тогда этот слой гомеоморфен либо $S^{2}$, либо $T^{2}$ ввиду условия на кривизну многообразия и параболичности слоения. Если это тор, то он плоский, но тогда $M^{3} \backslash P$ содержит открытое множество. Поэтому ввиду насьщенности $M^{3} \backslash P$ и аналитичности метрики $M^{3}$ плоское. В случае же, когда слой 
гомеоморфен $S^{2}$, в силу теоремы стабильности Риба заключаем, что $M^{3}$ гомеоморфно $S^{2} \times S^{1}$, и слоение есть тривиальное $S^{2}$-расслоение над окружностью.

Случай 2. Пусть $\mathscr{F}$ не имеет компактных слоев. Если хотя бы один слой плоский, то $M^{3}$ плоское (см. выше). В противном случае слои имеют неотрицательную секционную кривизну и содержат точки, где кривизна больше 0. Ввиду теоремы 5 все слои гомеоморфны плоскости. Но тогда из теоремы 4 следует, что $M^{3}$ гомеоморфно тору. Значит, в силу неотрищательности секционной кривизны $M^{3}$ снова плоское. Таким образом, теорема В доказана.

\section{4. Примеры.}

ПримеР 1 (негеодезируемые слоения на плоском торе). Напомним, что слоение на многообразии $M$ негеодезируемо, если оно не является вполне геодезическим ни для какой метрики $g$ на $M$.

Пусть $\mathbb{R}^{3}$ - трехмерное линейноепространство со стандартной евклидовой метрикой. Определим 1-форму на $\mathbb{R}^{3}$ в декартовых координатах $(x, y, z)$ :

$$
\omega(x)=h(x) d x+(1-h(x)) d y
$$

где $h(x)$ - любая гладкая функция, удовлетворяющая следующим условиям:

1) $0 \leqslant h(x) \leqslant 1$;

2) $h(x)$ периодическая с периодом 1 ;

3) $h(1 / 2)=0$;

4) $h(1 / 2-x)=h(1 / 2+x)$

5) $h(0)=h(1)=1$;

6) $h(x)$ возрастает на $[1 / 2,1]$.

Нетрудно видеть, что $\omega \wedge d \omega=0$. Поэтому $\omega$ задает слоение коразмерности 1 в силу теоремы Фробениуса. Так как слои являются цилиндрическими поверхностями, это слоение - параболическое. Очевидно, что слоение инвариантно относительно свободного действия группы $\mathbb{Z}^{3}$ на $\mathbb{R}^{3}$, определяемого параллельными переносами на векторы с целочисленными координатами. Поэтому, профакторизовав $\mathbb{R}^{3}$ по $\mathbb{Z}^{3}$, мы получим параболическое слоение на плоском торе $T^{3}$. Это слоение имеет единственный компактный слой, диффеоморфный тору, которьй является вполне геодезическим подмногообразием в $T^{3}$. Полученное слоение неориентируемо, однако его двулистное накрытие является уже ориентируемьм слоением на $T^{3}$. Оно имеет два компактных слоя. Взяв на накрытии индуцированную метрику, получим ориентируемое параболическое слоение на плоском торе. Оно не является геодезируемым. Это следует из того, что всякая траектория, ортогональная слоению и стартующая с компактного слоя, ни разу больше на него не попадает [10]. Заметим, что существуют также примеры негеодезируемых параболических слоений и на других плоских пространственных формах.

ПримеР 2 (параболическоеслоение на трехмерной сфере $S^{3}$ ). На $S^{3}$ существует метрика класса $C^{\infty}$ неотрицательной секционной кривизны такая, что слоение Риба является параболическим для этой метрики.

Рассмотрим следующую параметризацию единичной сферы $S^{3}$ в цилиндрических координатах $(t, \varphi, \theta)$ :

$$
x=t \cos \varphi, \quad y=t \sin \varphi, \quad z=\sqrt{1-t^{2}} \cos \theta, \quad \tau=\sqrt{1-t^{2}} \sin \theta .
$$


Особенностями данной параметризации являются две зацепленные окружности $t=0$ и $t=1$. Определим следующую метрику на $S^{3}$ :

$$
d s^{2}=d t^{2}+g^{2}(t) d \varphi^{2}+f^{2}(t) d \theta^{2},
$$

где $g$ и $f$ - выпуклые неотрицательные функции, определяемые следуюшим образом. Возьмем произвольные положительные константы $a, A_{1}$ и $A_{2}$. Положим

$$
G(x)= \begin{cases}a \frac{\alpha\left(A_{2}-x\right)}{\alpha\left(A_{2}-x\right)+\alpha\left(x-A_{1}\right)}, & A_{1} \leqslant x \leqslant A_{2}, \\ 0, & x \geqslant A_{2} .\end{cases}
$$

Здесь

$$
\alpha(x)= \begin{cases}e^{-1 / t}, & t>0 \\ 0, & t \leqslant 0 .\end{cases}
$$

Константы $a, A_{1}$ и $A^{2}$ можно выбрать так, чтобы выполнялись условия

$$
A_{1}<A_{2}<1-\frac{1}{\sqrt{2}}, \quad \int_{0}^{A_{2}} G(x) d x=1 .
$$

Теперь положим

$$
g(t)=\int_{0}^{t} G(x) d x .
$$

Нетрудно видеть, что $g \in C^{\infty}$ и $g^{\prime \prime}(t) \leqslant 0$, т.е. $g$ - вьпуклая функция. Теперь положим по определению $f(t)=g(1-t)$. Ввиду особого выбора метрики вблизи сингулярностей параметризации метрика определена глобально. Построенная метрика есть метрика неотрицательной секционной кривизны, причем лишь при $A_{1}<A_{2}$ кривизна не равна 0 тождественно. Определим слоение Риба на $S^{3}$. Пусть $(t, \varphi)$ - цилиндрические координаты на $\mathbb{R}^{3}$. Определим 1 -форму на $\mathbb{R}^{3}$ следующим образом: $\omega=h(t) d t+(1-h(t)) d \theta$, где $h(t)$ определяется в виде

$$
h(t)=\left\{\begin{array}{l}
0, \quad 0 \leqslant t \leqslant A_{2}, \\
h(t) \text { возрастает на }\left[A_{2}, 1 / \sqrt{2}\right], \\
1, \quad t \geqslant 1 / \sqrt{2} .
\end{array}\right.
$$

Нетрудно убедиться, что $\omega \wedge d \omega=0$. Поэтому форма $\omega$ задает слоение на $\mathbb{R}^{3}$. Ограничив его на область $t \leqslant 1 / \sqrt{2}$, а затем соответственно профакторизовав, получим слоение на полнотории. Склеив стандартньм образом два таких полнотория, получим слоение на $S^{3}$. Убедимся, что полученное слоение параболическое для построенной вьше метрики. Действительно, в области $0 \leqslant t \leqslant A_{2}$ имеем метрическое произведение слоя $\left\{\theta=\right.$ const, $\left.0 \leqslant t \leqslant A_{2}\right\}$ на окружность $t=0$. Следовательно, в этой области слоение является вполне геодезическим. По аналогичньм рассуждениям слоение вполне геодезическое в области $1-A_{2} \leqslant t \leqslant 1$. В области $A_{2}<t<1-A_{2}$ метрика плоская и имеет вид $d s^{2}=d t^{2}+d \varphi^{2}+d \theta^{2}$. Заметим, что через каждую точку этой области проходит одна из окружностей, принадлежащая некоторому слою: $\{t=$ const, $\theta=$ const $\}$, если $A_{2}<t \leqslant 1 / \sqrt{2}$, или $\{t=$ const, $\varphi=$ const $\}$, если $1 / \sqrt{2} \leqslant t<1-A_{2}$. Однако эти окружности являются геодезическими в $S^{3}$, поэтому $K^{e} \leqslant 0$ в этой области. Ввиду теоремы А и неотрицательности кривизны метрики следует, что $K^{e}=0$ в этой области. Заметим, что единственньм вполне геодезическим слоем является плоский тор, отвечающий параметру $t=1 / \sqrt{2}$. 
ПРИМЕР 3 (сильногиперболическое слоение на расслоении единичных касательных векторов над пространством постоянной отрицательной кривизны, равной -1$)$. Пусть $T_{1} P$ - расслоение единичных касательных векторов над пространством постоянной отрицательной кривизны, равной -1 . Рассмотрим геодезический поток на $P$. Он задает орисферическое слоение на $T_{1} P$. Если рассмотреть изометрическое накрытие $p: T_{1} H^{2} \rightarrow T_{1} P$, то прообразом слоя будет поверхность в $T_{1} H^{2}$, определяемая единичньм векторньм полем вдоль геодезических, параллельных (по Лобачевскому) некоторой фиксированной геодезической на $H^{2}$, т.е. геодезических, сходящихся к некоторой фиксированной точке $A$ на абсолюте.

Рассмотрим на $H^{2}$ параболическую систему координат $(r, t)$, где координатным линиям, отвечающим параметру $r$, соответствует пучок геодезических, определенньй выше, а координатньм линиям, соответствующим параметру $t$, отвечают орициклы, определяемые как орбиты однопараметрической группы поворотов вокруг точки $A$. В этой системе координат метрика на $H^{2}$ имеет вид $d s^{2}=d r^{2}+e^{-2 r} d t^{2}$. Метрическая связность, соответствующая этой метрике, имеет вид:

$$
\Gamma^{1}=\left(\begin{array}{cc}
0 & 0 \\
0 & e^{2 r}
\end{array}\right), \quad \Gamma^{2}=\left(\begin{array}{cc}
0 & -1 \\
-1 & 0
\end{array}\right) .
$$

Рассмотрим метрику Сасаки на $T_{1} H^{2}$. Пусть $\left(x^{i}, \xi^{i}\right)=\left(r, t, \xi^{1}, \xi^{2}\right)$ - локальные координаты на $T_{1} H^{2}$, где в качестве ортонормированного базиса $\left(\xi^{1}, \xi^{2}\right)$ можно взять единичные касательные векторные поля вдоль координатных линий $r, t$. Тогда в этих координатах метрика имеет вид

$d \sigma^{2}=d s^{2}+g_{i k} D \xi^{i} D \xi^{k}=d r^{2}+e^{-2 r} d t^{2}+\left(d \xi^{1}+e^{2 r} \xi^{2} d t\right)^{2}+e^{-2 r}\left(d \xi^{2}-\xi^{1} d t-\xi^{2} d r\right)^{2}$, где $D \xi^{i}=d \xi^{i}+\Gamma_{j k}^{i} \xi^{j} d x^{k}(i, k=1,2)$.

Из того, что движения перестановочны с параллельным переносом, а также из конкретного вида метрики Сасаки, следует, что изометрическое накрытие $r: H^{2} \rightarrow P$ поднимается до изометрического накрытия $p: T_{1} H^{2} \rightarrow T_{1} P$. Поэтому в локальных координатах $\left(r, t, \xi^{1}, \xi^{2}\right)$ метрика на $T_{1} P$ совпадает с метрикой $d \sigma^{2}$ на $T_{1} H^{2}$.

УТВЕРЖДЕНИЕ. Пусть $T_{1} P$ - сферическое расслоение к пространству постоянной кривизны, равной -1, с сасакиевой метрикой. Тогда орисферическое слоение на $T_{1} P$ является сильногиперболическим и гармоническим, причем слои имеют постоянную секиионную кривизну -1 и постоянную внешнюю кривизну $-1 / 4$.

ДокАЗАТЕЛЬСТво. Пусть $\mathscr{L}$ - произвольный слой орисферического слоения на $T_{1} P$. Выберем локальную систему координат так, чтобы $\mathscr{L}$ локально задавался в виде поверхности $\varphi(r, t)=(r, t, 1,0)$. Тогда касательные к координатньм линиям векторы имеют вид $\varphi_{r}(1,0,0,0)$ и $\varphi_{t}(0,1,0,0)$. Для доказательства данного утверждения необходимо доказать следующую лемму.

Лемма. Индуиированная метрика на $\mathscr{L}$ из $T_{1} P$ имеет вид $d q^{2}=d r^{2}+2 e^{-2 r} d t^{2}$, т.е. является метрикой постоянной кривизны, равной -1 .

ДоказАТЕльство. Так как локально $\mathscr{L}$ задается как поверхность $\varphi(r, t)=(r, t, 1,0)$, имеем $d \xi^{1}=0$. Из конкретного вида связности $(2)$ следует, что $D \xi^{1}=d \xi^{1}+\Gamma_{j k}^{1} \xi^{j} d x^{k}=0$. Из аналогичных соображений следует, что $\xi^{2}=d \xi^{2}=0$ и $D \xi^{2}=\Gamma_{12}^{2} d x^{2}=-d t$. Поэтому индуцированная метрика $d q^{2}$ имеет вид $d q^{2}=d r^{2}+2 e^{-2 r} d t^{2}$. Лемма доказана. 
Пусть $\left(e_{1}, e_{2}, e_{3}\right)$ - локальный ортонормированньй базис в точке $(x, v)$ многообразия $T_{1} P$, причем $e_{1}$ - горизонтальный лифт $v, e_{2}$ - горизонтальный лифт $v^{\perp}\left(v^{\perp} \perp v\right)$, а $e_{3}$ - вертикальньй лифт $v^{\perp}$. Напомним, что горизонтальный лифт вектора $Z=\left(Z^{i}\right)$ в точку $(x, \xi)$ - это вектор $Z^{H}=\left(Z^{i},-\Gamma_{j k}^{i} Z^{j} \xi^{k}\right)$, а вертикальньй лифт - это вектор $Z^{V}=\left(0,0, Z^{i}\right)(i, j, k, l=1,2)$. Определим теперь базис $\left(e_{1}, e_{2}, e_{3}\right)$ в точке $(r, t, 1,0)$ :

$e_{1}=(1,0,0,0)$ - горизонтальный лифт вектора $\xi^{1}=(1,0)$;

$e_{2}=\left(0, e^{r}, 0, e^{r}\right)$ - горизонтальньй лифт вектора $\xi^{2}=\left(0, e^{r}\right)$;

$e_{3}=\left(0,0,0, e^{r}\right)$ - вертикальный лифт вектора $\xi^{2}=\left(0, e^{r}\right)$.

Разложим векторы $\varphi_{r}=(1,0,0,0)$ и $\varphi_{t}=(0,1,0,0)$ по базису $\left(e_{1}, e_{2}, e_{3}\right): \varphi_{r}=e_{1}$ и $\varphi_{t}=e^{-r}\left(e_{2}-e_{3}\right)$. Отождествив $T_{1} P$ с расслоением реперов над $P$, можно показать, что структурные уравнения на $T_{1} P$ имеют следующий вид:

$$
d \Theta_{1}=-\Theta_{2} \wedge \Theta_{3}, \quad d \Theta_{2}=\Theta_{1} \wedge \Theta_{3}, \quad d \Theta_{3}=\Theta_{1} \wedge \Theta_{2}
$$

где $\left(\Theta_{1}, \Theta_{2}, \Theta_{3}\right)$ - базис ковекторов, двойственньй к $\left(e_{1}, e_{2}, e_{3}\right)$. С другой стороны, $d \Theta_{i}=-\sum_{j} \Theta_{j} \wedge w_{i j}$, где $w_{i j}-$ компоненты формы метрической связности. Сравнивая эти системы, получим $w_{12}=\frac{3}{2} \Theta_{3}, w_{13}=\frac{1}{2} \Theta_{2}, w_{23}=-\frac{1}{2} \Theta_{1}$. Теперь, учитьвая что рассматриваемое слоение определяется формой $\Theta_{2}+\Theta_{3}$, нетрудно убедиться, что в базисе $\left(e_{1},\left(e_{2}-e_{3}\right) / \sqrt{2}\right)$, касательном к слоению, вторая квадратичная форма слоения постоянна и имеет вид

$$
A=\left(\begin{array}{cc}
0 & -1 / 2 \\
-1 / 2 & 0
\end{array}\right)
$$

откуда и следует утверждение.

Автор выражает благодарность профессору А. А. Борисенко за научное руководство, а также Ю. А. Николаевскому за полезные советы в ходе подготовки данной работы.

\section{СПИСОК ЦИТИРОВАННОЙ ЛИТЕРАТУРЫ}

[1] Asimov D. Average Gaussian curvature of leaves of foliations // Bull. Amer. Math. Soc. 1978. V. 84. P. 131-133.

[2] Brito F., Langevin R., Rosenberg H. Intégrales de courbure sur des variétés feuilletées // J. Differential Geom. 1981. V. 16. P. 123-135.

[3] Tondeur Ph. Foliations on Riemannian Manifolds. Universitext. New York: Springer, 1988.

[4] Rosenberg R. Foliations by planes // Topology. 1968. V. 7. P. 131-138.

[5] Cheeger J., Gromoll D. On the structure of complete manifolds of nonnegative curvature // Ann. of Math. 1972. V. 96. P. 413-443.

[6] Борисенко А. А. О цилиндрических многомерных поверхностях в пространстве Лобачевского // Укр. геом. сб. 1990. Т. 33. С. 18-27.

[7] Аминов Ю. А. Геометрия векторного поля. М.: Наука, 1990.

[8] Maltz R. The nullity spaces of the curvature operator // Cahiers Topologie Géom. Différentielle Catégoriques. 1968. V. 8. P. 1-20.

[9] Eberlein P. Euclidean de Ram factor of a lattice of nonpositive curvature // J. Differential Geom. 1983. V. 18. P. 209-220.

[10] Johnson D., Witt L. Totally geodesic foliations // J. Differential Geom. 1980. V. 15. P. 225-235. 